\title{
Knockdown of KRT17 by siRNA induces antitumoral effects on gastric cancer cells
}

\author{
Mihaela Chivu-Economescu ${ }^{1}$ (D) Denisa L. Dragu ${ }^{1} \cdot$ Laura G. Necula $^{1,2,3}$. \\ Lilia Matei $^{1}$ - Ana Maria Enciu ${ }^{3,4}$ - Coralia Bleotu ${ }^{1}$ Carmen C. Diaconu ${ }^{1}$
}

Received: 12 October 2016/Accepted: 4 March 2017/Published online: 15 March 2017

(C) The International Gastric Cancer Association and The Japanese Gastric Cancer Association 2017

\begin{abstract}
Background Keratin 17 (KRT17) was shown to be an important molecular marker for predicting the carcinogenesis, progression, and prognosis of various cancer types. Our previous studies identified KRT17 as a possible biomarker for gastric cancer by gene microarray, with an elevated expression that occurred early during tumorigenesis and increased during tumor progression. Based on these findings, we aimed to investigate KRT17 biological functions in gastric adenocarcinoma and its possible use as a rational molecular target for anticancer therapy.

Methods We used RNA interference-mediated knockdown of KRT17 expression and analyzed the effects on cell proliferation, cell migration, and signal transduction in two gastric cell lines (AGS and NCI-N87) in vitro and on xenograft growth in vivo.

Results The functional analysis of KRT17 knockdown cell lines showed a decreased cell proliferation (with $42.36 \% \pm 3.2 \%$ ) and migration ability (with $37.2 \% \pm 6.2 \%$ ) relative to scrambled siRNA control. The in vivo tumorigenicity on nude mice exhibited a significant decrease in tumor weight with $69.14 \%$ in xenografts
\end{abstract}

Mihaela Chivu-Economescu

mihaela.chivu@gmail.com; mihaela.chivu@virology.ro

1 Cellular and Molecular Department, Stefan S. Nicolau Institute of Virology, Mihai Bravu 285 Ave,

030304 Bucharest, Romania

2 Nicolae Cajal Institute, Titu Maiorescu University, Bucharest, Romania

3 Biochemistry-Proteomics Department, Victor Babes National Institute of Pathology, Bucharest, Romania

4 Cell Biology and Histology Department, Carol Davila University of Medicine and Pharmacy, Bucharest, Romania obtained from AGS cells and $84.43 \%$ in xeno-NCI-N87 tumors. The analysis on KRT17 knockdown outcome on intracellular signaling identifies AKT/mTOR as the main affected pathway that sustains proliferation and survival, and also the AMPK $\alpha 1 /$ CREB pathway that was recently shown to induce organ protection and antiinflammatory response.

Conclusions Our results highlight KRT17 as a possible biomarker in gastric cancer promoting tumor growth, motility, and invasion, and suggest that KRT17 can be a valuable molecular target for development of anti-gastric cancer-specific therapies.

Keywords Keratin 17 . Biomarkers - Gastric cancer . Targeted therapy $\cdot$ RNA interference

\section{Introduction}

Gastric (stomach) cancer is the fifth most common malignancy in the world, with more than $70 \%$ of cases occurring in developing countries, and the third leading cause of cancer death worldwide ( 723,000 deaths; $8.8 \%$ of the total) [1].

Systemic therapy for these tumors is based primarily on cytotoxic chemotherapy with 5-fluorouracil alone, or in combination with a second platinum or non-platinum active drug, or as triple therapy (i.e., in combination with a third chemotherapeutic drug), but its effectiveness is limited by drug resistance [2]. Furthermore, a substantial proportion of gastric cancer patients who have undergone curative surgery develop recurrent disease. Five-year relative survival varied between $10.6 \%$ and $24.0 \%$ [3], based on cancer stage and localization. Protection of patients from drug side effects and the need for a personalized 
treatment are currently major concerns in the gastric cancer drug development field. Therefore, it remains an important clinical need to identify new cancer molecular markers as new targets for gastric adenocarcinoma therapy.

In a previous study aimed to understand gradual biological variations during gastric tumorigenesis, we identified keratin 17, also known as cytokeratin 17 (KRT17, K17), among other candidate genes that are involved in tumor progression and metastasis. KRT17 was the most overexpressed gene (fold change, 95.69), with an elevated expression that occurred early and increased by 5.66 fold during tumor progression [4].

Keratins are 10-nm filaments that form a framework within the cellular cytoplasm protecting cells from mechanical and nonmechanical agents. Keratins have also been recognized as regulators of other cellular properties and functions, including apicobasal polarization, motility, cell size, protein synthesis, and membrane traffic and signaling [5]. KRT17 is associated with hyperproliferation, and is induced, along with keratin 6 (K6) and keratin 16 (K16), early after acute injury of human skin, such as that occurring during wound healing, in the palmoplantar epidermis, and in the hair follicles [6]. Keratin 17, although not present in normal mature epithelia, is expressed in stem cells of embryonic ectoderm, skin appendages, and the endocervical mucosa, and is reexpressed in carcinomas [7].

Keratins are extensively used as diagnostic tumor markers in adenocarcinomas, as epithelial malignancies largely maintain the specific keratin patterns associated with their respective cells of origin [8]. Until the present, KRT17 has been used as diagnostic marker in tumor pathology for breast cancer $[9,10]$ and skin squamous carcinoma [8]. Keratins have also been recognized as prognostic indicators in a variety of epithelial malignancies such as breast tumors [9] and epithelial ovarian cancer [11]. Furthermore, KRT17 expression was found to be significantly increased in cervical squamous cell carcinoma [12] and gastric cancer [13], being significantly associated with poor survival.

Several studies have provided evidence for active keratin involvement in cancer cell invasion and metastasis, as well as in treatment responsiveness, and have set the foundation for further exploration of the role of keratins as multifunctional regulators of epithelial tumorigenesis [14].

Considering previous findings, we hypothesized that the selective downregulation of KRT17 expression by RNA interference (RNAi)-mediated knockdown represents a reasonable therapeutic strategy for the treatment of gastric adenocarcinoma. Therefore, we silenced KRT17 and analyzed the effects on cell proliferation, cell migration, and signal transduction in two gastric cell lines (AGS and NCIN87) in vitro and on xenograft tumor growth in vivo.

\section{Materials and methods}

\section{Tissue samples}

Gastric adenocarcinoma tissue samples and adjacent nontumor tissue were collected from patients during surgery at the Center of General Surgery and Liver Transplantation of Fundeni Clinical Institute, after written informed consents and approval of the Ethical Committee were obtained. The tissues were frozen in liquid nitrogen immediately after excision and then stored at $-70{ }^{\circ} \mathrm{C}$. The gastric cancer samples were classified according to the American Joint Committee on Cancer TNM (tumor, node, and metastasis) staging system. Two TNM stage IV samples and their corresponding normal tissue were selected for Western blot analysis, and also two tumor samples, TNM stages III and II, respectively.

\section{Cell culture}

Cells from the human gastric adenocarcinoma cell line AGS (catalogue no. CRL-1739) and NCI-N87 (catalogue no. CRL-5822) were purchased from American Type Culture Collection (USA). AGS cells were cultured in Ham's F12 medium (Sigma Aldrich) supplemented with $10 \%$ fetal bovine serum (Biochrom) at $37{ }^{\circ} \mathrm{C}$ in $5 \% \mathrm{CO}_{2}$. The NCI-N87 cells were cultured in RPMI 1640 medium (Sigma Aldrich) under the same culture conditions.

\section{Small interfering RNAs transfection}

Small interfering RNAs (siRNA) targeting the KRT17 transcript were purchased from Thermo Scientific. Sequences were $\left(5^{\prime}-3^{\prime}\right)$ : GCCGAGGAUUGGUUCUUCATT and CGUCAGGUGCGUACCAUUG. To determine the effects of siRNA delivery, a scrambled siRNA (silencer negative control, AM4636; Thermo Scientific) which has no significant sequence similarity and does not target human gene sequences was used. For KRT17 knockdown, the AGS and NCI-N87 cells were seeded in six-well plates at $6 \times 10^{5}$ cells/well. After $24 \mathrm{~h}$ incubation at $37^{\circ} \mathrm{C}$, the cells were transfected with $10 \mathrm{nM}$ specific KRT17 siRNAs or scrambled siRNA using siPORT NeoFX Transfection Agent and serum-free OptiMEM (both from Thermo Scientific). Complete medium with $10 \%$ fetal bovine serum was added until $2.5 \mathrm{ml} /$ well final volume.

\section{Quantitative real-time (RT) polymerase chain reaction $(\mathrm{PCR})$ analysis}

Cells were harvested $48 \mathrm{~h}$ after transfection, and total RNA was extracted with Tri Reagent (Sigma Aldrich, St. Louis, 
MO, USA). For each sample, $2 \mu \mathrm{g}$ RNA was used for cDNA synthesis with Access Quick RT-PCR kit (Promega). Quantitative RT-PCR reactions were performed on an ABI 7300 real-time PCR machine (Applied Biosystems) using the TaqMan gene expression assay from Thermo Scientific: Hs01588578_m1 (KRT17), Hs01075862_m1 (CD44), and Hs00173626_m1 (VEGFA). The data were normalized to the housekeeping gene $18 \mathrm{~S}$ transcripts, and the relative mRNA expression between the KRT17 siRNA-treated and Scr siRNA-treated cells was calculated using the $2^{-\mathrm{ddCt}}$ method.

\section{Cell proliferation and cell-cycle analyses}

The cells were seeded in 96-well culture plates at a density of $6 \times 10^{3}$ cells/well and treated with $10 \mathrm{nM}$ siRNA. The CellTiter 96 Aqueous One Solution (Promega) assay was performed to determinate the gene downregulation effect on cell proliferation at 48,72 , and $96 \mathrm{~h}$ after transfection. For each well, $20 \mu \mathrm{L}$ MTS was added and the plates were maintained $4 \mathrm{~h}$ at $37{ }^{\circ} \mathrm{C}$. The absorbance was measured at $550 \mathrm{~nm}$ using a TECAN GENios reader. All experiments were effectuated in triplicate. Values were calculated as mean and SD of percentage change induced by each siRNAs as compared to control cells. For the cell-cycle assay, the cells treated with siRNA for $72 \mathrm{~h}$ were fixed in $70 \%$ cold ethanol for at least $30 \mathrm{~min}$ at $-20{ }^{\circ} \mathrm{C}$. After washing twice in phosphate-buffered saline (PBS), cells were incubated $15 \mathrm{~min}$ at $37{ }^{\circ} \mathrm{C}$ with RNAse A $(1 \mathrm{mg} / \mathrm{ml})$ and $1 \mathrm{~h}$ with propidium iodide $(100 \mu \mathrm{g} / \mathrm{ml})$. The acquisition used a Epics Beckman Coulter flow cytometer. Data were analyzed using FlowJo software and expressed as fractions of cells in the different cell-cycle phases.

\section{Cell migration assays}

The migration capacity of the transfected cells was evaluated by two methods: the scratch wound-healing method and QCM chemotaxis 96-well cell migration assay (Millipore). For the scratch wound-healing method, the cells were cultured in 6-well plates at a $6 \times 10^{5}$ cells/dish density, then treated with $10 \mathrm{nM}$ KRT17 or scrambled siRNAs. After $24 \mathrm{~h}$, transfected cells were mechanically disrupted with a sterile $100-\mu \mathrm{l}$ tip. The wound-healing images were acquired at point 0 and after $24 \mathrm{~h}$ with an inverted Zeiss Axio Observer D1 microscope. For the QCM chemotaxis 96-well cell migration assay, after $48 \mathrm{~h}$ from transfection, cells were starved for $6 \mathrm{~h}$, trypsinized, and resuspended at a density of $3 \times 10^{5}$ cells/well in serumfree medium in the $8.0-\mu \mathrm{m}$ dishes inserted in a 24 -well plate. In the lower part of the well complete medium containing $10 \%$ fetal bovine serum was added. After maintaining the system for $24 \mathrm{~h}$ at $37{ }^{\circ} \mathrm{C}\left(5 \% \mathrm{CO}_{2}\right)$, the cells that had migrated to the lower part of the insert were enzymatically detached, lysed, and stained for 15 min with DNA-binding dye, CyQuant GR.
Quantification was achieved by reading fluorescence at 480/520 nm (excitation/emission) using a Wallac Victor 2 spectrophotometer (Perkin Elmer). Values were calculated as mean and SD of percentage reduction induced by each siRNA as compared to control cells.

\section{Matrigel invasion assay}

Invasion assays were performed with the trans-well FluoroBlok Tumor Invasion System (BD Biosciences) using 24-well inserts with an 8.0- $\mu$ m-pore-size PET membrane coated with matrigel. The plate was rehydrated, and DMEM/ FBS $5 \%(\mathrm{v} / \mathrm{v})$ was added to the lower chamber. In the upper chamber, $5 \times 10^{4}$ siRNA-transfected cells, labeled with $10 \mu \mathrm{g} / \mathrm{ml} \mathrm{DiIC}_{12}(3)$, a fluorescent dye, were added in $400 \mu \mathrm{l}$ serum-free DMEM. The plates were incubated for $20 \mathrm{~h}$ at $37{ }^{\circ} \mathrm{C}$ and $5 \% \mathrm{CO}_{2}$. Quantification of cell invasion was achieved by measuring the fluorescence of invading cells at $549 / 565 \mathrm{~nm}$ using a Wallac Victor 2 spectrophotometer (Perkin Elmer). Images were acquired using an inverted Zeiss Axio Observer D1 microscope. Values were calculated as mean and SD of percentage reduction induced by each siRNA as compared to control cells.

\section{Protein profiler analysis}

A human phospho-kinase (ARY003B; R\&D Systems) array was used to analyze the levels of phosphorylated kinases according to the manufacturer's protocol. The kit allows simultaneous analysis of the 43 kinase phosphorylation sites and 2 related total proteins. Briefly, for immunoblot analysis, $1 \times 10^{6}$ cells grown in 6-well plates were transfected with KRT17 siRNA or Scr siRNA and harvested in lysis protein buffer after $48 \mathrm{~h}$. A quantity of $400 \mu \mathrm{g}$ total proteins diluted in array buffer were incubated with the nitrocellulose membranes at $4{ }^{\circ} \mathrm{C}$ overnight. Subsequently, the membranes were washed to remove unbound proteins and incubated with a cocktail of biotinylated antibodies for $4 \mathrm{~h}$. After repeated washing, the arrays were incubated with a streptavidin-HRP solution, and the signals were detected using the chemiluminescent reaction. The signals were captured using MicroChemi 4.2 (Bio Imaging Systems), and the images were analyzed using ImageJ 1.42 software (National Institute of Health) after subtraction of background levels (negative control) from sample signal levels and normalization to positive control signal to allow comparison between samples. Experiments were performed twice.

\section{Western blot analysis}

Whole protein extracts were obtained using T-PER Tissue Protein Extraction Reagent (78510; Thermo Fisher, USA) 
supplemented with Complete O, Mini, EDTA-free Protease Inhibitor Cocktail (Roche Applied Science), and the concentration of total proteins was determined using BCA Protein Assay Reagent (Pierce). Whole protein extracts $(40 \mu \mathrm{g})$ were electrophoretically separated by SDS-PAGE and transferred onto PVDF membranes that were subsequently blocked in Tris-buffer saline (TBS)-0.5\% Tween 20 with $2 \%$ bovine serum albumin and then incubated with the primary antibodies against proteins of interest at $4{ }^{\circ} \mathrm{C}$ overnight. The antibodies used (1:1000 dilutions) were mouse monoclonal anti-cytokeratin 17 clone CK-E3 (Sigma Aldrich), mouse monoclonal anti-phospho-p38 MAPK (Thr180/Tyr182) antibody clone 28B10 (Cell Signaling Technology), rabbit monoclonal anti-p38 MAPK antibody clone D13E1 (Cell Signaling Technology), rabbit monoclonal anti-phospho-JNK1 + JNK2 pThr183 + pTyr185 antibody clone D12H7L17 (Invitrogen), mouse monoclonal anti-JNK1 + JNK2 antibody clone 279Q38 (Invitrogen), mouse monoclonal anti-ERK1/ ERK2 antibody clone 216703 (RD Systems), rabbit monoclonal anti-phospho-ERK1 (T202/Y204)/ERK2(T185/ Y187) antibody clone 269434 (RD Systems), rat monoclonal anti-mTOR antibody (RD Systems), rabbit polyclonal anti-phospho-mTOR antibody (RD Systems), rabbit monoclonal anti-phospho-Akt (Ser473) (193H12) (Cell Signaling Technology), rabbit monoclonal anti-Akt (pan) (11E7) (Cell Signaling Technology), mouse monoclonal anti-beta-catenin antibody (RD System), rabbit anti-phospho- $\beta$-catenin (S33/S37) antibody (RD Systems), and mouse monoclonal anti- $\beta$-actin clone Ac-74 (Sigma Aldrich). Proteins of interest were detected with the appropriate secondary antibodies (1:2000 dilutions) conjugated with HRP: anti-rat IgG (Thermo Fischer), antirabbit IgG (RD Systems), and anti-mouse (RD Systems). Signals were developed using ECL HRP chemiluminescent substrate (Invitrogen) and captured using a MicroChemi 4.2 system (Bio Imaging Systems).

\section{In vivo studies}

For in vivo experiments, 6-week-old CD1 athymic nude mice grown in our husbandry were used. The original breed stock was purchased from Charles River Laboratories and held under standardized pathogen-free conditions. The study was approved by the Ethics Committee of Stefan S. Nicolau Institute of Virology. For each cell line, the mice $(n=32)$ were divided in four groups: a control group $(n=8)$ and a KRT17 siRNA group for each cell line. The 6-week-old CD1 athymic nude mice were subcutaneously injected into the dorsal flank with a 0.2-ml mixture of $1 \times 10^{6}$ transfected cells and Matrigel (BD Biosciences). Nine weeks after injection, the mice were killed and the tumor size and weight were recorded.

\section{Statistical analysis}

All data were analyzed using GraphPad Prism 5.0 software. Values are represented as mean \pm standard deviation (SD). The statistical significance of differences was determined by Student's $t$ test or the two-factor analysis of variance (ANOVA). A $p$ value $<0.05$ was considered to be statistically significant.

\section{Results}

\section{Downregulation of KRT17 expression by target siRNA transfection}

Previously we showed that KRT17 mRNA expression was found elevated in gastric adenocarcinoma samples respective to normal tissue and that its level increased by tumor stage. The results were confirmed at protein level, where KRT17 was found expressed by human gastric adenocarcinoma samples of various tumor stages (Fig. 1a).

To evaluate the implication of KRT17 in gastric cancer cell proliferation and migration, and on their tumorigenic capacity, KRT17 expression was inhibited in the AGS and NCI-N87 cell lines using RNA interference technology. The qPCR results confirmed the downregulation of KRT17 gene expression in an effective and constant manner at $10 \mathrm{nM}$ KRT17 siRNA. The transfection induced a significant downregulation on KRT17 transcript levels with $70-74 \%$ on both cell lines compared with control siRNAtransfected cells $(p<0.001)$ (Fig. 1b). Western blot analysis showed a reduction of KRT17 protein expression with $56.28 \%$ and $69.57 \%$, respectively, as quantified using Image J software (Fig. 1c).

KRT17 knockdown allowed us to analyze the biological effects of the targeted gene transcription by investigating the effects induced on signaling pathways, and also on proliferation and migration capacities of gastric adenocarcinoma cells.

\section{Effect of KRT17 siRNA knockdown on cell proliferation and cell cycle}

The downregulation of KRT17 gene expression decreased proliferation in both AGS and NCI-N87 cell lines when compared with nontreated and scrambled siRNA-transfected cells. An inhibitory effect was noted mainly at $72 \mathrm{~h}$ after transfection, when cell proliferation decreased significantly with $40.54 \% \pm 3.85 \%$ in AGS cells and with $24.49 \% \pm 0.67 \%$ in NCI-N87, respectively $(p<0.01)$ (Fig. 2a). Consistent with cell proliferation decrease, cellcycle analysis indicated that KRT17 knockdown increases $\mathrm{G}_{0} / \mathrm{G}_{1}$ phase with $11.58 \% \pm 0.63 \%$ and reduces S-phase 


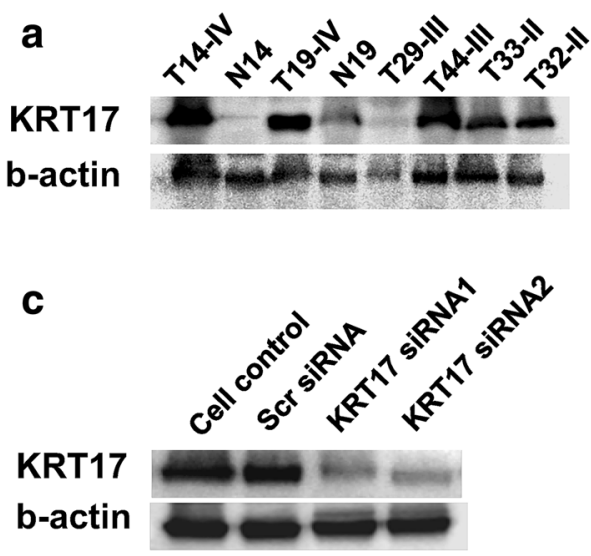

Fig. 1 Knockdown of KRT17 mRNA and protein expression in gastric adenocarcinoma cell lines. a Western blot analysis using proteins from human gastric adenocarcinoma tissues shows KRT17 protein expression in tumor samples of various stages. Two different TNM stage IV samples (14 and 19) and their corresponding normal tissue, and also two tumor samples TNM stages III (29 and 44) and II (33 and 32), were used for western blot analysis. b Quantitative real-

cell population with $14.55 \% \pm 1.15 \%$ (Fig. 2b). No significant results were seen for NCI-N87 cells.

\section{KRT17 siRNA knockdown decrease cell motility and invasion capacity}

The KRT17 influence on cell migration was evaluated using two methods: the scratch wound-healing method and a QCM Chemotaxis 96-well cell migration assay. The obtained results showed that downregulation of KRT17 gene expression leads to a significant decrease of cell motility in both cell lines (Fig. 3a). In comparison to the AGS cell line in which cell migration was decreased by $29.71 \% \pm 3.27 \%$ on average versus control, in the NCIN87 cell line the migration decreased by $71.70 \% \pm 4.20 \%$ relative to the Scr siRNA-transfected control (Fig. 3b).

The hallmark of metastatic cells is their capacity to invade through the basement membrane and migrate all over the body. For that, cells must be able both to secrete proteases that digest the basement membrane as well as to migrate to be invasive. For testing this ability, a tumor invasion system based on an 8.0- $\mu \mathrm{m}$-pore-size PET membrane that has been coated with matrigel matrix (BD Falcon FluoroBlok 24-Multiwell Insert Plate) was used. siRNA-transfected cells were labeled with a DilC ${ }_{12}$, and quantitation of cell invasion was performed $24 \mathrm{~h}$ later by measuring the fluorescence of invading cells. Results showed that KRT17 downregulation induced a reduction of AGS cell line invasiveness by $28.09 \% \pm 2.50 \%$ versus the siRNA control. In contrast, NCI-N87 cell line invasion capacity decreased significantly, by $40.83 \% \pm 4.22 \%$ $(p<0.01)$ (Fig. 3c, d). b

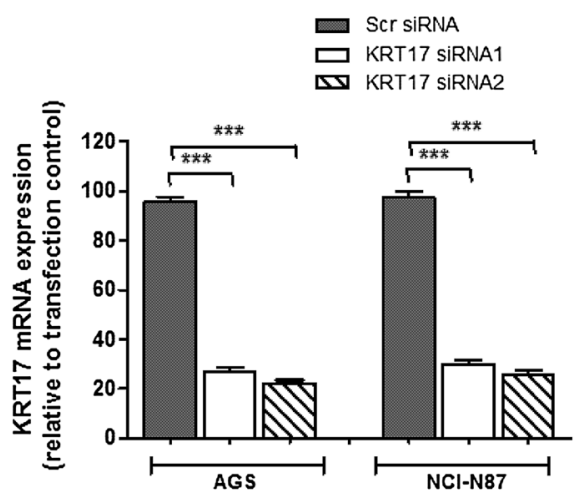

time PCR analysis of KRT17 mRNA levels. Knockdown of KRT17 inhibits the gene expression in gastric adenocarcinoma AGS and NCIN87 cell lines. Data represent three independent transfection experiments. Values are represented as mean $\pm \mathrm{SD}$. $* * * p<0.001$ vs. scrambled siRNA clone (negative control). $\mathbf{c}$ Western blot analysis of KRT17 siRNAs transfected cells demonstrate efficient KRT17 protein knockdown

\section{Analysis of KRT17 knockdown outcome on intracellular signaling pathways}

To evaluate the effects of KRT17 knockdown on major signaling pathways, the phosphorylation profiles of the main involved kinases were analyzed using a human phosphokinase array. The significant alterations in protein expression and activation after KRT17 knockdown are presented in Fig. 4a, b. Our results demonstrated that downregulation of KRT17 decreases the phosphorylation of AKT and mTOR, which participate in the main signaling pathway involved in proliferation and survival. Also, our results showed an increase in phosphorylation level of several kinases included in the MAPK pathway: ERK1/2, JNK $1 / 2 / 3$, and RSK $1 / 2 / 3$, and also of the AMPK $\alpha 1 /$ CREB pathway that was recently shown to trigger an antiinflammatory response. Moreover, in the AGS cell line, KRT17 siRNA treatment induced an increased phosphorylation of Chk-2, p53, and p27 kinases that induce cell-cycle arrest in accordance with cell proliferation decrease. Those results were confirmed by Western blot analysis in cells treated with KRT17 and control siRNA (Fig. 4c). From all explored proteins, $\beta$-catenin showed a particularly interesting behavior, presenting an increased level in AGS cell line after treatment and with no significant changes in NCIN87 cells.

\section{Downregulation of KRT17 retards tumor growth in vivo}

To investigate the in vivo effect of KRT17 downregulation, $1 \times 10^{6}$ transfected cells, with siRNA KRT17 or Scr 
Fig. 2 Cell proliferation and cell-cycle analyses.

a Downregulation of KRT17 inhibits cancer cell proliferation on AGS and NCI-N87 cell lines. Cell proliferation was determined using CellTiter96 assay and recording absorbance at $550 \mathrm{~nm}$ with a

spectrophotometric plate reader. Data represent three

independent experiments.

Values are represented as mean \pm SD. $* * p<0.01$.

b Cell-cycle phase progression of AGS cells treated with control siRNA and KRT17 siRNAs. The strong decrease in the S-phase population for AGS cells after KRT17 knockdown is visible in the plots
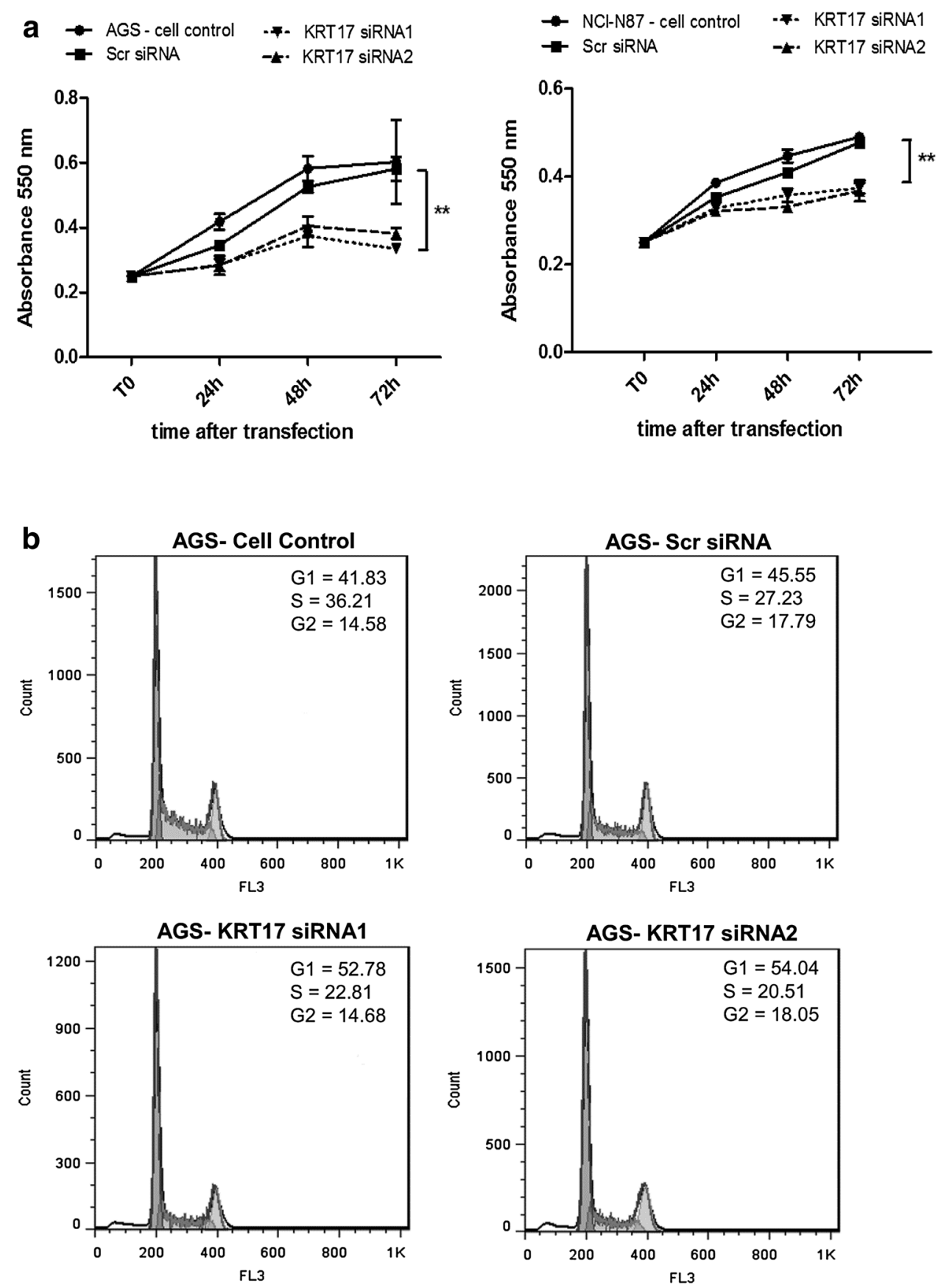

siRNA control, were inoculated subcutaneously in immunodeficient mice. At 9 weeks post injection, mice were killed and tumors were collected.

Tumor size and weight were significantly lower in KRT17 siRNA transfected cells than those treated with Scr siRNA (Fig. 5a, b). KRT17 knockdown decreased tumor weight mean significantly, with $69.14 \%$ in xenografts obtained from the AGS cell line and with $84.43 \%$ in xenoNCI-N87 tumors.

In the light of positive results regarding tumor growth inhibition, we thought next to investigate the residual level of KRT17 in xenografts at 9 weeks post injection by quantitative real-time PCR. Surprisingly, the results indicated the maintenance of the KRT17 gene downregulation in both cell lines, with $50.75 \% \pm 12.25 \%$ in AGS and $69.03 \% \pm 2.49 \%$ in NCI-N78 cells, compared with the levels from Scr siRNA-treated cells. The initial downregulation of KRT17 gene before inoculation was 70-74\%. The mRNA levels of two additional genes were tested: VEGFA and CD44. Those genes were chosen because of their impact on vascularization and cell migration, both important features in the tumorigenesis process. The results 

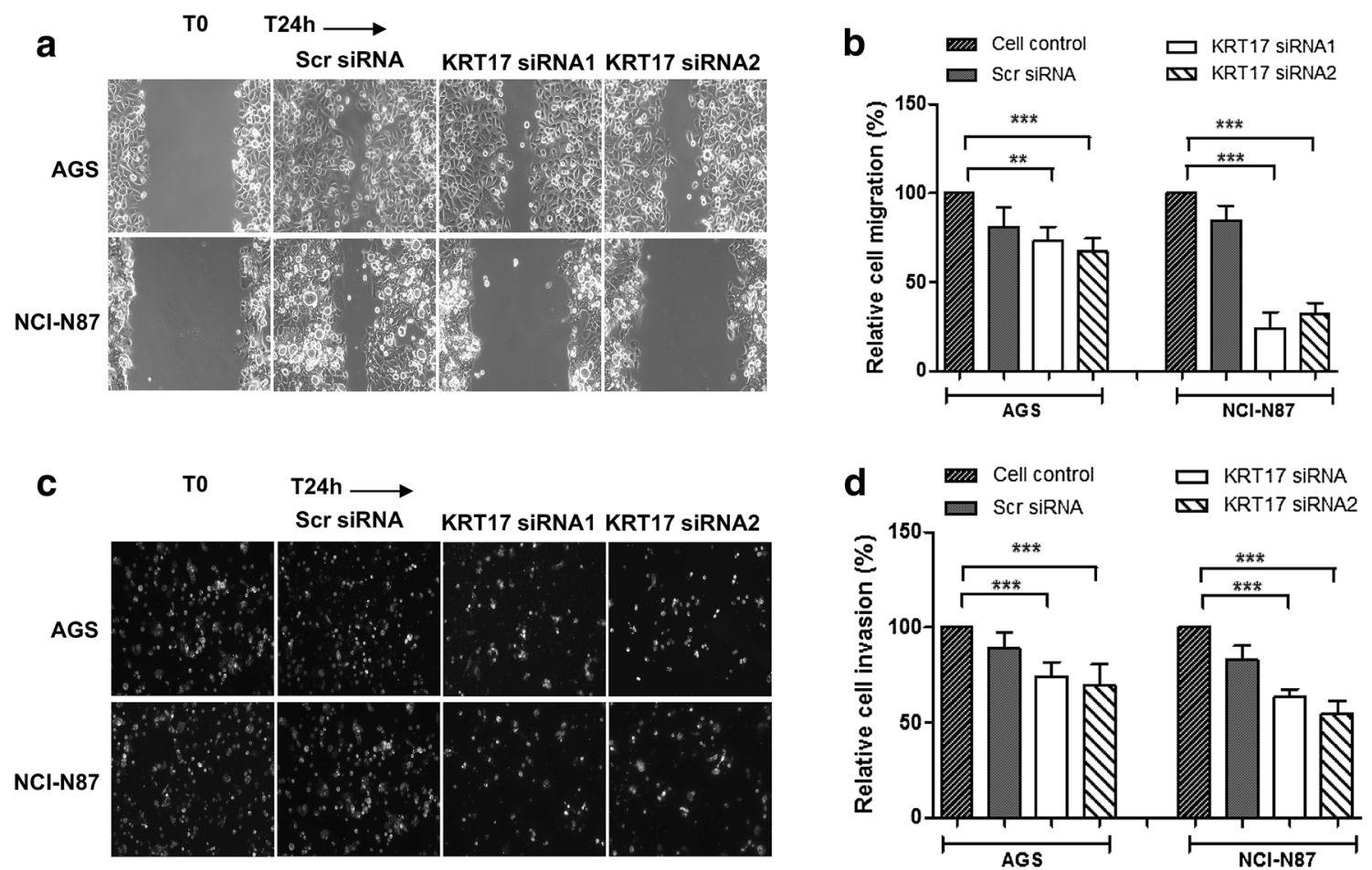

Fig. 3 KRT17 knockdown reduced gastric adenocarcinoma cell migration and invasion capacity in vitro. a Scratch assay. Confluent monolayers of siRNA-transfected cells were mechanically disrupted with a sterile $100-\mu 1$ tip, then photographed immediately (T0) and at $24 \mathrm{~h}$ after scratching. Phase contrast $\times 200$. b Cell motility assay using QCM Chemotaxis 96-well test from Millipore/Chemicon:

showed that the VEGF mRNA level decreased with $53.1 \% \pm 20.72 \%$ in AGS xenografts and with $59.54 \pm 18.96 \%$ in NCI-N87 compared with the levels from Scr siRNA-treated cells (Fig. 5c). For CD44, the mRNA levels decreased with $59.54 \% \pm 18.96 \%$ for AGS and with $82.84 \% \pm 1.83 \%$ for NCI-N87, respectively, which could explain the reduced tumor weight, as well as the decrease in cell motility and invasion capacity noted in previous experiments.

\section{Discussion}

Although important advances were made in understanding the molecular biology of gastric cancer, this aggressive solid tumor continues to have a poor prognosis and few efficacious therapeutic options. Because of the heterogeneity of gastric cancer it is difficult to make substantial progress in its therapy, and an important remaining clinical need is to identify and validate new molecular markers for future clinical approach in cancer treatment.

Our former study highlighted the KRT17 gene that appears to meet the criteria of a good biomarker for monitoring gastric cancer progression, as well as for early quantification of motility by reading fluorescence at $480 / 520 \mathrm{~nm}$. c Matrigel invasion of DilC $_{12}$-labeled nontargeted and KRT17targeted cells. $\times 200$. d Quantification of fluorescence from invading cells at 549/565 $\mathrm{nm}$. Data represent two independent experiments performed in triplicate. Values are represented as mean $\pm \mathrm{SD}$. $* * p<0.01 ; * * * p<0.001$

detection [4]. These results confirmed previous findings that identified KRT17 as a diagnostic and prognostic marker for breast tumors [9], epithelial ovarian cancer [11], cervical squamous cell carcinoma [12], and gastric cancer, wherein KRT17 was positively associated with tumor tissue by immunohistochemical staining [13]. These findings imply an oncogenic role of KRT17, but its exact mechanism of action remains unclear.

The aim of this study was to investigate KRT17 biological function in gastric adenocarcinoma and its possible use as a target for anticancer therapy. For this purpose, we used RNA interference, which has been proved to be a powerful tool in finding and validating new targets for anticancer therapy. However, the key for success is to find a gene that is expressed in cancer cells but not in normal cells. According to the study by Troy et al., KRT17 meets this criteria, not being present in normal mature epithelia, excepting endocervical mucosa and skin appendages, including hair, glands, and nails, and being reexpressed in carcinomas [7].

Our results showed that selective KRT17 knockdown by RNA interference could significantly suppress cell proliferation, inhibit the cell migration and invasion of gastric adenocarcinoma cells in vitro, and reduce tumor growth in vivo. Interesting, the in vivo experiments revealed that 
a AGS - Scr SIRNA

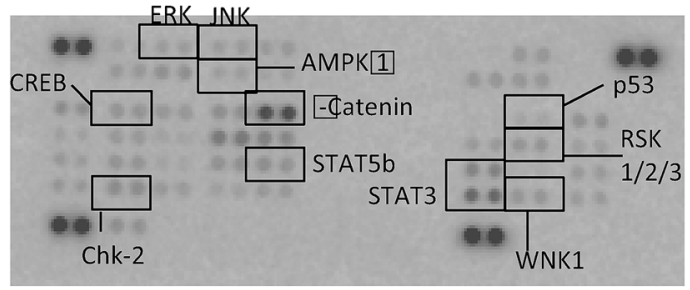

\section{AGS - KRT17 siRNA}

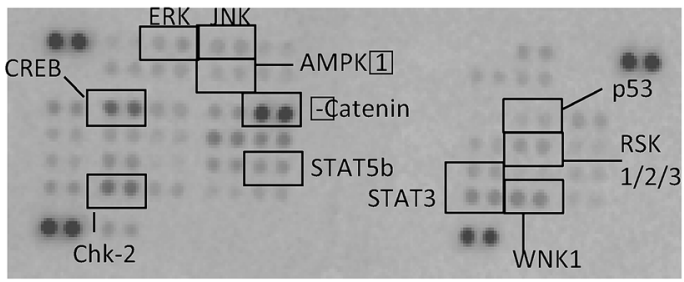

b

AGS

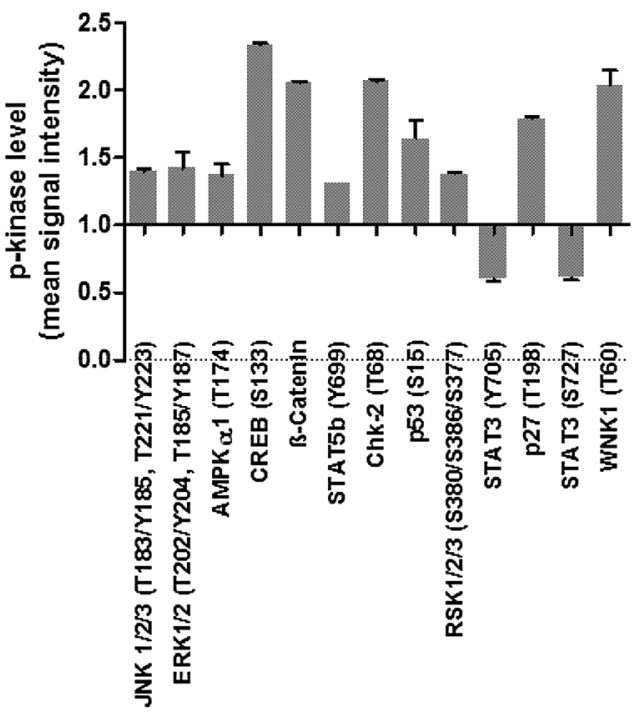

\section{NCI-N87 - Scr siRNA}

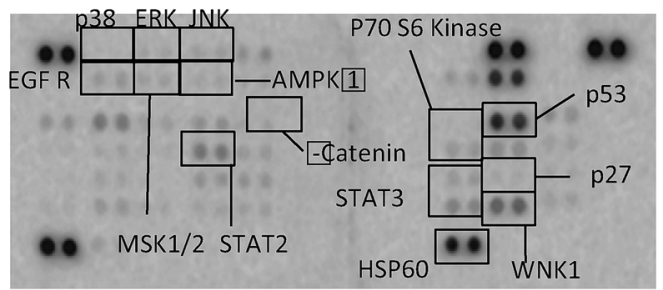

NCI-N87 - KRT17 SiRNA

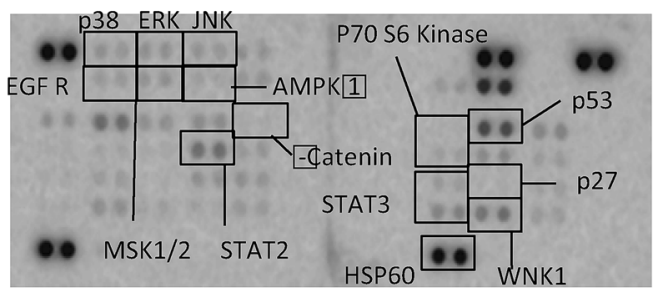

$\mathrm{NCl}-\mathrm{N} 87$

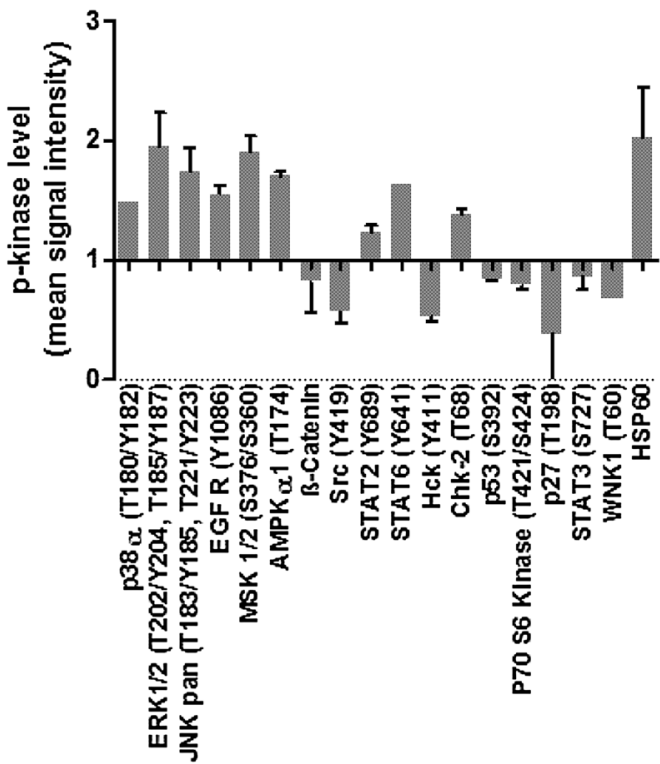

C

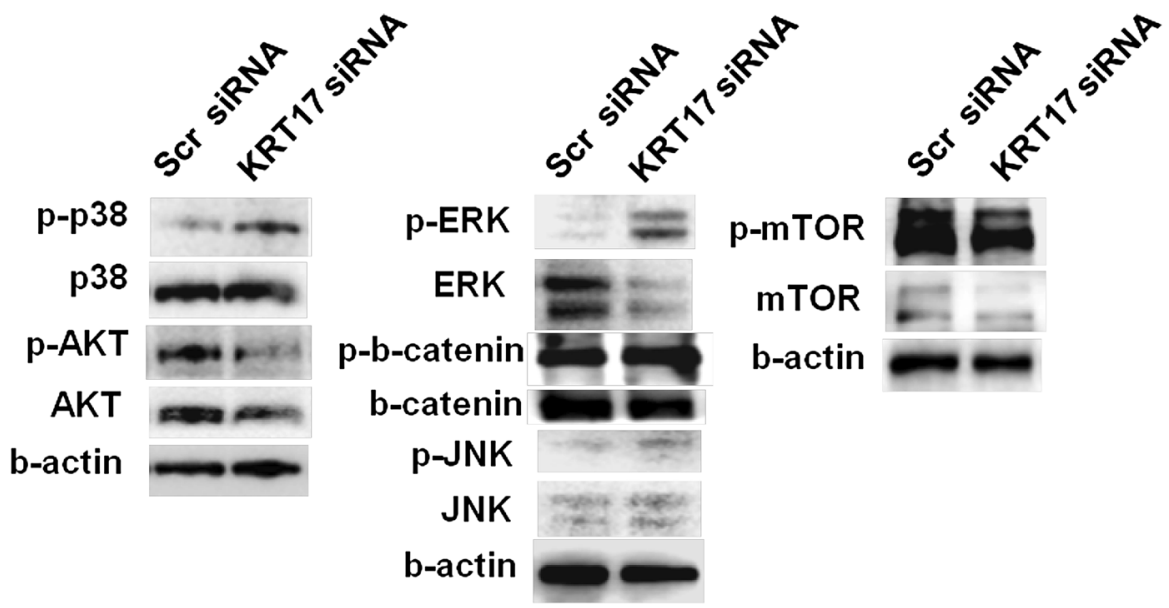


4Fig. 4 Knockdown of KRT17 decreases p38 and AKT/mTOR signaling and activates ERK/JNK MAPK. a Proteome profiler analysis: representative images of the chemiluminescence signals with differences in phosphokinase levels. The kinases indicated in black boxes are those with significant modified level (representative experiment). b Densitometric analysis of the proteome profiler blots depicting kinases with significant changes $(p<0.05)$ in mean phosphorylation level after transfection with KRT17 siRNA relative to Scr siRNA treatment. Data represent two independent experiments. c Western blot analysis was performed at $48 \mathrm{~h}$ post transfection of scramble and KRT17 siRNAs. Specific antibodies and b-actin were used for detection of indicated proteins (NCI-N87 cell line is presented: a representative experiment)

KRT17 expression was maintained at a low level in xenografts during 9 weeks post injection. We presume that this long-acting effect results from the method of pretreating cells with siRNA in vitro and injecting them after $48 \mathrm{~h}$, when the cell proliferation rate was already diminished. Thus, gene silencing was less affected by siRNA dilution from cell division. Previous studies reported that the duration of gene silencing in low/non-dividing cells could be extended at 3-4 weeks for in vivo injected naked siRNA, which is a more unstable system than the one we used [15, 16]. Additionally, two important genes were found to be downregulated, VEGFA and CD44. Those genes have a major impact on vascularization and cell migration, both important features in the tumorigenesis process. The obtained results could explain the diminution of tumor weight as well as the decrease in cell motility and invasion capacity noted in cell culture experiments. The analysis on KRT17 knockdown outcome on intracellular signaling pathways reveals a decrease in the phosphorylation of AKT and mTOR, which participate in the main signaling pathway involved in proliferation and survival. The frequent activation of $\mathrm{PI} 3 \mathrm{~K} / \mathrm{AKT} / \mathrm{mTOR}$ in gastric cancer is increasingly recognized since the identification of mutations in PI3KCA gene. Our results are in agreement with previous data published by Sankar et al., who demonstrated that KRT17 regulates cellular adhesion by activating AKT/PKB (protein kinase B) signaling and induces oncogenic transformation in Ewing sarcoma as an upregulated target of glioma-associated oncogene homologue 1. Another study published by Kim et al. indicates KRT17 as having the leading role in regulating epithelial cell size and growth by binding to the adaptor protein 14-3$3 \sigma$ and stimulating the mTOR pathway, thus regulating protein synthesis [6].

In addition, our results showed that KRT17 knockdown induces an increase in the phosphorylation levels of Chk-2, p53, and p27 involved in cell-cycle arrest. These findings were available only for AGS cells, which are highly proliferating cells derived from an advanced undifferentiating adenocarcinoma. Chk-2 is a serine/threonine kinase activated in response to double-strand DNA breaks. Following its activation, Chk2 phosphorylates downstream effectors such as p53 and E2F1, which further leads to initiation of DNA repair or the sustained block of cell-cycle progression and induces apoptosis. Chk2 is known to act as a p53 kinase, phosphorylating the $\mathrm{N}$-terminal activation domain of p53 [17]. p27 is a cyclin-dependent kinase inhibitor acting as a tumor suppressor by preventing cellcycle progression. A decrease of p27 expression was reported in gastric cancer and it was associated with metastasis in patients with gastric carcinoma [18, 19]. Our findings on p27-induced phosphorylation and the decrease of cell proliferation after KRT17 knockdown in gastric adenocarcinoma cells comes as a reflection of the observation by Escobar-Hoyos et al. of an inverse correlation between KRT17 and p27 $7^{\mathrm{KIP} 1}$ expression. They conducted an excellent study on the potential role of KRT17 in mediating cancer cell-cycle progression and tumor growth in human cervix, breast, and pancreatic cancer cells, and revealed that KRT17 is translocated in the nucleus where it is bound specifically to the $\mathrm{p} 27^{\mathrm{KIP} 1}$ tumor suppressor and promotes its nuclear export and subsequent degradation in the cytoplasm of tumor cells [20].

Another positive effect of KRT17 knockdown was the activation of adenosine monophosphate-activated protein kinase (AMPK) and AMP response element-binding protein (CREB) by Ser133 phosphorylation. This is an important feature as recent studies are sustaining the positive effect of AMPK-CREB pathway activation as a novel protection mechanism against organ injury and systemic inflammation by inducing the expression of cytoprotective genes including manganese superoxide dismutase (MnSOD) and heme oxygenase-1 (HO-1) [21, 22].

A secondary effect of the KRT17 siRNA treatment was the activation of the MAPK pathway, revealed through increased phosphorylation of ERK1/2 and JNK 1/2/3 in both cell lines. Additionally, a part of the ERK5 MAP kinase pathway, WNK1 and RSK1/2/3, is activated on the AGS cell line, whereas p38 kinase is activated on NCI-N87 cells. Unfortunately, this inverse correlation is a wellknown side effect of PI3K/AKT/mTOR pathway downregulation. Rexer et al. [23] and Turke et al. [24] also demonstrated this correlation, showing that the blockade of MEK, downstream substrate of the MAPK pathway, resulted in feedback activation of the PI3K/AKT pathway, but the combined inhibition of both PI3K and MEK synergistically inhibited the growth of breast cancer cells. Consequently, Shi et al. adopted a new approach demonstrating that simultaneously targeting of the RAS/MAPK and PI3K/AKT by silencing of the K-RAS and AKT2 oncogenes may inhibit pancreatic cancer cell growth [25]. Collectively, these data sustain a novel approach in developing antitumor therapy, suggesting the necessity of 
a

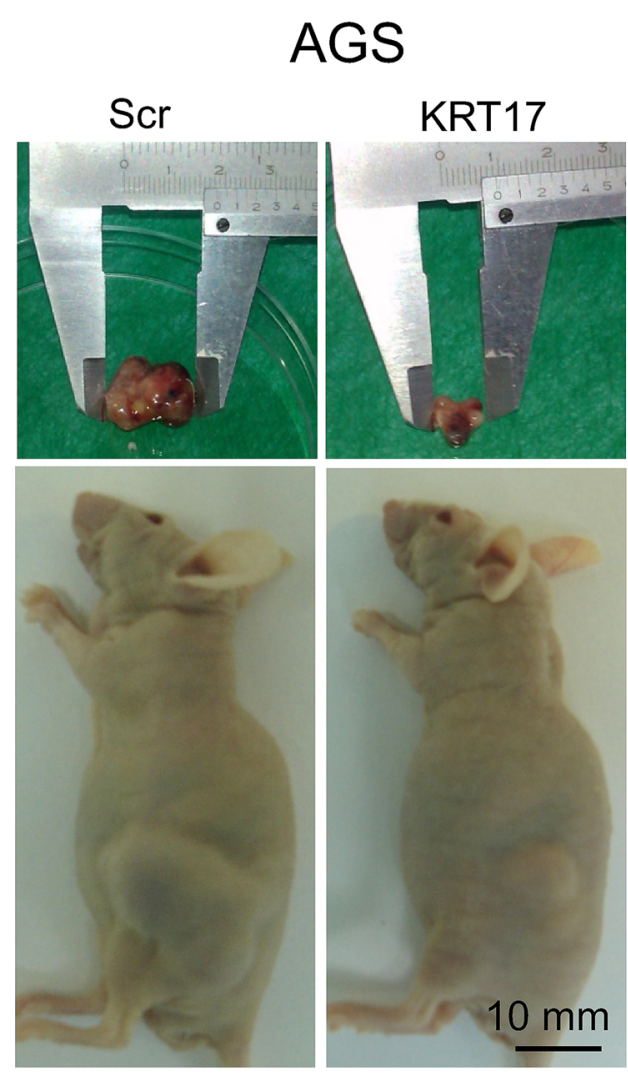

b
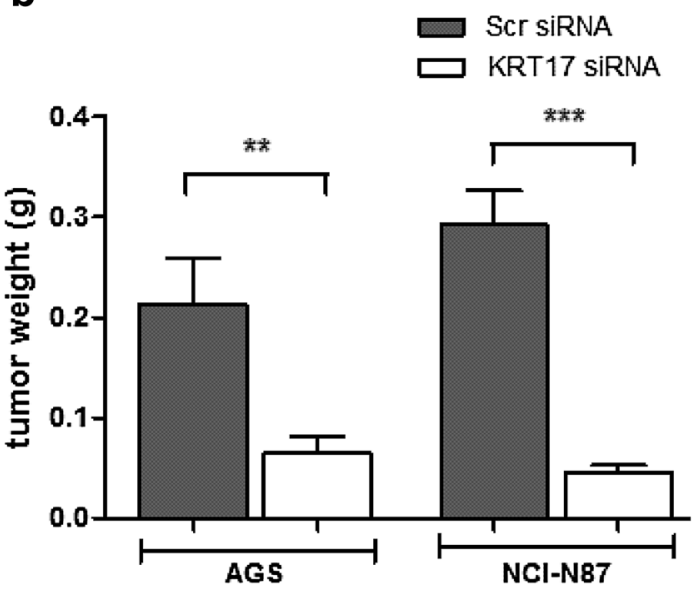

Fig. 5 Downregulation of KRT17 inhibits the growth of AGS- and NCI-N87-derived xenografts in athymic nude mice. a Representative macrographic images of mice and tumor xenografts from each group ( $n=8$ per group). b Tumor weight $(\mathrm{g})$ in mice after 9 weeks post inoculation. c KRT17 knockdown was maintained after 9 weeks in

using inhibitors that target both MAPK and AKT pathways.

One interesting feature of our results was $\beta$-catenin behavior. The Wnt/b-catenin pathway is well known for promoting cancer cell proliferation and invasion through loss of cell adhesion and increased cell migration [26]. When the pathway is active, $\beta$-catenin accumulates and

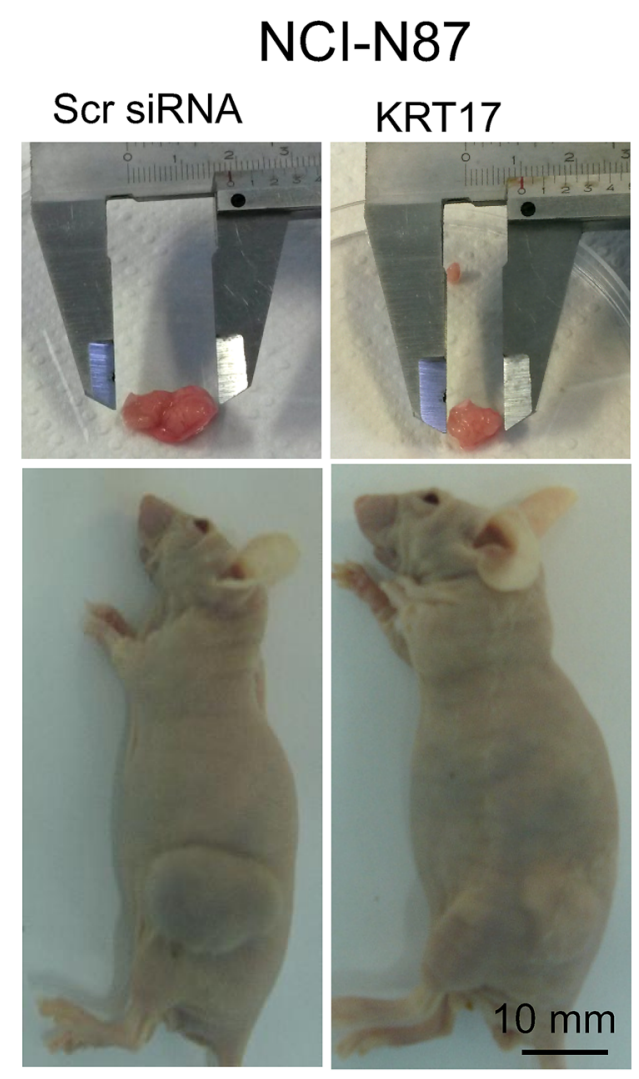

\section{C}

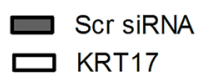

ㅁII VEGFA

$\$ \infty \times 14$

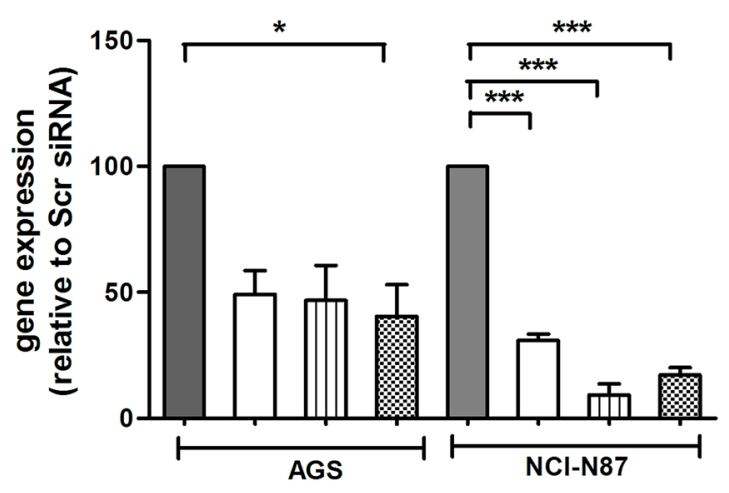

xenografts and influences the expression of genes related to vascularization (VEGFA) and migration (CD44). Values are represented as mean $\pm \mathrm{SD}$. $* p<0.05, * * p<0.01, * * * p<0.001$ vs. scrambled siRNA clone (negative control)

travels to the nucleus where it binds to TCF/LEF and activates the expression of various genes, such as CD44, c-Myc, and cyclin D [27]. We observed an increased level of $\beta$-catenin in the AGS cell line after siRNA treatment and no significant changes in NCI-N87 cells. We suggest that this disparity results from differences between the two cancer cells lines used: AGS is a fast proliferating cell line 
and was derived from an advanced undifferentiated adenocarcinoma, in contrast with NCI-N87, which originated from a well-differentiated gastric adenocarcinoma. Our results showed that the KRT17 siRNA treatment successfully blocked cell proliferation and cell-cycle progress of aggressive growing cells, such as AGS or advanced undifferentiated carcinomas, but had a modest effect in reducing their motility and invading capacity. On NCI-N87 cells, KRT17 downregulation had a minor effect on cell proliferation ability but a better outcome in inhibiting migration and invasion sustained by a significantly reduced CD44 gene expression that is found among genes targeted and regulated by the Wnt pathway [28].

\section{Conclusion}

The analysis of KRT17 knockdown outcome on intracellular signaling identifies two main affected pathways: AKT/mTOR, that sustains proliferation and survival, and the AMPK $\alpha 1 /$ CREB pathway, which was recently shown to induce organ protection and antiinflammatory response. Additionally, a decrease in phosphorylation of Chk-2, p53, and p27 kinases involved in cell-cycle arrest was noticed in the fast proliferating AGS cell line. These findings highlight KRT17 as a possible biomarker and molecular target for development of anti-gastric cancer-specific therapy. Additionally, a screening for possible detection of KRT17 in serum samples from patients with gastric cancer and a correlation between its level and tumor progression and/or response to therapy will be very useful. Its detection and possible linkage with the tumorigenic process will bring valuable help in close monitoring of disease progression, prognoses, and response to specific treatments as a noninvasive screening tool.

Acknowledgements This study was supported by structural funds from POS CCE O2.2.1. 433/2012, UEFISCDI PCCA PN II 133/2012 and OPERATIONAL COMPETITIVITATY PROGRAM 2014-2020 POC-A1-A1.1.4-E-2015/P_37_798/149/2016.

\section{Compliance with ethical standards}

Conflict of interest The authors declare that they have no conflict of interest.

Animal studies All institutional and national guidelines for the care and use of laboratory animals were followed.

\section{References}

1. Ferlay J, Soerjomataram I, Dikshit R, Eser S, Mathers C, Rebelo $\mathrm{M}$, et al. Cancer incidence and mortality worldwide: sources, methods and major patterns in GLOBOCAN 2012. Int J Cancer. 2015;136:359-86.
2. Xu ZY, Tang JN, Xie HX, Du YA, Huang L, Yu PF, et al. 5-Fluorouracil chemotherapy of gastric cancer generates residual cells with properties of cancer stem cells. Int J Biol Sci. 2015;11:284-94.

3. Bouvier AM, Sant M, Verdecchia A, Forman D, Damhuis R, Willem Coebergh $\mathrm{J}$, et al. What reasons lie behind long-term survival differences for gastric cancer within Europe? Eur J Cancer. 2010;46:1086-92.

4. Chivu Economescu M, Necula LG, Dragu D, Badea L, Dima SO, Tudor S, et al. Identification of potential biomarkers for early and advanced gastric adenocarcinoma detection. Hepatogastroenterology. 2010;57:1453-64.

5. Karantza V. Keratins in health and cancer: more than mere epithelial cell markers. Oncogene. 2011;30:127-38.

6. Kim S, Wong P, Coulombe PA. A keratin cytoskeletal protein regulates protein synthesis and epithelial cell growth. Nature (Lond). 2006;441:362-5.

7. Troy TC, Turksen K. Commitment of embryonic stem cells to an epidermal cell fate and differentiation in vitro. Dev Dyn. 2005;232:293-300.

8. Moll R, Divo M, Langbein L. The human keratins: biology and pathology. Histochem Cell Biol. 2008;129:705-33.

9. van de Rijn M, Perou CM, Tibshirani R, Haas P, Kallioniemi O, Kononen J, et al. Expression of cytokeratins 17 and 5 identifies a group of breast carcinomas with poor clinical outcome. Am J Pathol. 2002;161:1991-6.

10. Sorlie T, Perou CM, Tibshirani R, Aas T, Geisler S, Johnsen H, et al. Gene expression patterns of breast carcinomas distinguish tumor subclasses with clinical implications. Proc Natl Acad Sci USA. 2001;98:10869-74.

11. Wang YF, Lang HY, Yuan J, Wang J, Wang R, Zhang XH, et al. Overexpression of keratin 17 is associated with poor prognosis in epithelial ovarian cancer. Tumour Biol. 2013;34:1685-9.

12. Escobar-Hoyos LF, Yang J, Zhu J, Cavallo JA, Zhai H, Burke S, et al. Keratin 17 in premalignant and malignant squamous lesions of the cervix: proteomic discovery and immunohistochemical validation as a diagnostic and prognostic biomarker. Mod Pathol. 2014;27:621-30.

13. Ide M, Kato T, Ogata K, Mochiki E, Kuwano H, Oyama T. Keratin 17 expression correlates with tumor progression and poor prognosis in gastric adenocarcinoma. Ann Surg Oncol. 2012;19:3506-14.

14. Obermajer N, Doljak B, Kos J. Cytokeratin 8 ectoplasmic domain binds urokinase-type plasminogen activator to breast tumor cells and modulates their adhesion, growth and invasiveness. Mol Cancer. 2009;8:88.

15. Bartlett DW, Davis ME. Insights into the kinetics of siRNAmediated gene silencing from live-cell and live-animal bioluminescent imaging. Nucleic Acids Res. 2006;34:322-33.

16. Omi K, Tokunaga $\mathrm{K}$, Hohjoh $\mathrm{H}$. Long-lasting RNAi activity in mammalian neurons. FEBS Lett. 2004;558:89-95.

17. Bartek J, Lukas J. Chk1 and Chk2 kinases in checkpoint control and cancer. Cancer Cell. 2003;3:421-9.

18. Wiksten JP, Lundin J, Nordling S, Kokkola A, von Boguslawski K, Haglund C. The prognostic value of p27 in gastric cancer. Oncology. 2002;63:180-4.

19. Calik M, Demirci E, Altun E, Calik I, Gundogdu OB, Gursan N, et al. Clinicopathological importance of Ki-67, p27, and p53 expression in gastric cancer. Turk J Med Sci. 2015;45:118-28.

20. Escobar-Hoyos LF, Shah R, Roa-Pena L, Vanner EA, Najafian N, Banach A, et al. Keratin-17 promotes p27KIP1 nuclear export and degradation and offers potential prognostic utility. Cancer Res. 2015;75:3650-62.

21. Thornton CC, Al-Rashed F, Calay D, Birdsey GM, Bauer A, Mylroie H, et al. Methotrexate-mediated activation of an AMPKCREB-dependent pathway: a novel mechanism for vascular 
protection in chronic systemic inflammation. Ann Rheum Dis. 2016;75:439-48.

22. Escobar DA, Botero-Quintero AM, Kautza BC, Luciano J, Loughran P, Darwiche S, et al. Adenosine monophosphate-activated protein kinase activation protects against sepsis-induced organ injury and inflammation. J Surg Res. 2015;194:262-72.

23. Rexer BN, Ghosh R, Arteaga CL. Inhibition of PI3K and MEK: it is all about combinations and biomarkers. Clin Cancer Res. 2009; 15:4518-20.

24. Turke AB, Song Y, Costa C, Cook R, Arteaga CL, Asara JM, et al. MEK inhibition leads to PI3K/AKT activation by relieving a negative feedback on ERBB receptors. Cancer Res. 2012;72:3228-37.
25. Shi XH, Liang ZY, Ren XY, Liu TH. Combined silencing of K-ras and Akt2 oncogenes achieves synergistic effects in inhibiting pancreatic cancer cell growth in vitro and in vivo. Cancer Gene Ther. 2009;16:227-36.

26. Conacci-Sorrell M, Zhurinsky J, Ben-Ze'ev Ben-Ze'ev. The cadherin-catenin adhesion system in signaling and cancer. J Clin Invest. 2002;109:987-91.

27. MacDonald BT, Tamai K, He X. Wnt/beta-catenin signaling: components, mechanisms, and diseases. Dev Cell. 2009;17:9-26.

28. Marhaba R, Zoller M. CD44 in cancer progression: adhesion, migration and growth regulation. J Mol Histol. 2004;35:211-31. 